\title{
THE BEST BOUNDS IN GAUTSCHI-KERSHAW INEQUALITIES
}

\author{
Feng Qi, BAI-Ni GuO AND ChaO-PING CHEN
}

\begin{abstract}
By employing the convolution theorem of Laplace transforms, some asymptotic formulas and integral representations of the gamma, psi and polygamma functions, and other analytic techniques, this note provides an alternative proof of a monotonicity and convexity property by N. Elezović, C. Giordano and J. Pečarić in [4] to establish the best bounds in Gautschi-Kershaw inequalities. Moreover, some (logarithmically) complete monotonicity results on functions related to Gautschi-Kershaw inequalities are remarked.
\end{abstract}

Mathematics subject classification (2000): 33B15, 26A48, 26A51, 26D20.

Key words and phrases: monotonicity; convexity, gamma function; psi function, polygamma function, Gautschi-Kershaw inequality, (logarithmically) completely monotonic function.

\section{REFERENCES}

[1] M. Abramowitz, I. A. Stegun (Eds), Handbook of Mathematical Functions with Formulas, Graphs, and Mathematical Tables, National Bureau of Standards, Applied Mathematics Series 55, 4th printing, with corrections, Washington, 1965.

[2] C. BERG, Integral representation of some functions related to the gamma function, Mediterr. J. Math. 1, (4) (2004), 433-439.

[3] CH.-P. Chen, F. QI, The best bounds in Wallis' inequality, Proc. Amer. Math. Soc. 133, (2) (2005), $397-$ 401. RGMIA Res. Rep. Coll. 5, (4) (2002), Art. 13. URL: http://rgmia.vu.edu .au/v5n4 .html.

[4] N. Elezović, C. GIORDANO AND J. PeČARIĆ, The best bounds in Gautschi's inequality, Math. Inequal. Appl. 3, (2) (2000), 239-252.

[5] B.-N. GUO, F. QI, Inequalities for generalized weighted mean values of convex function, Math. Inequal. Appl. 4, (2) (2001), 195-202. RGMIA Res. Rep. Coll. 2, (7) (1999), Art. 11, 1059-1065. Available online at URL: http://rgmia.vu.edu.au/v2n7.html.

[6] W. Magnus, F. Oberhettinger And R. P. Soni, Formulas and Theorems for the Special Functions of Mathematical Physics, Springer, Berlin, 1966.

[7] F. QI, A note on Schur-convexity of extended mean values, Rocky Mountain J. Math., 35, (5) (2005), 1787-1793.

[8] F. QI, Generalized abstracted mean values, J. Inequal. Pure Appl. Math., 1, (1) (2000), Art. 4. Available online at URL: http://jipam.vu.edu.au/article.php?sid=97. RGMIA Res. Rep. Coll. 2, (5) (1999), Art. 4, 633-642. Available online at URL: http://rgmia.vu.edu.au/v2n5.html.

[9] F. QI, Generalized weighted mean values with two parameters, R. Soc. Lond. Proc. Ser. A Math. Phys. Eng. Sci. 454, (1978) (1998), 2723-2732.

[10] F. QI, Logarithmic convexity of extended mean values, Proc. Amer. Math. Soc., 130, (6) (2002), 1787-1796. RGMIA Res. Rep. Coll. 2, (5) (1999), Art. 5, 643-652. Available online at URL: http://rgmia.vu.edu.au/v2n5.html.

[11] F. QI, Monotonicity results and inequalities for the gamma and incomplete gamma functions, Math. Inequal. Appl. 5, (1) (2002), 61-67. RGMIA Res. Rep. Coll. 2, (7) (1999), Art. 7, 1027-1034. Available online at URL: http://rgmia.vu.edu.au/v2n7.html.

[12] F. QI, Schur-convexity of the extended mean values, RGMIA Res. Rep. Coll., 4, (4) (2001), Art. 4, 529-533. Available online at URL: http://rgmia.vu .edu.au/v4n4 .html. 
[13] F. QI, The extended mean values: definition, properties, monotonicities, comparison, convexities, generalizations, and applications, Cubo Mat. Educ. 5, (3) (2003), 63-90. RGMIA Res. Rep. Coll. 5, (1) (2002), Art. 5, 57-80. Available online at URL: http://rgmia.vu .edu .au/v5n1.html.

[14] F. QI, CH.-P. CHEN, A complete monotonicity property of the gamma function, J. Math. Anal. Appl. 296, (2) (2004), 603-607.

[15] F. QI, B.-N. GuO, Complete monotonicities of functions involving the gamma and digamma functions, RGMIA Res. Rep. Coll., 7, (1) (2004), Art. 8, 63-72. Available online at URL: http://rgmia.vu.edu.au/v7n1.html.

[16] F. QI, B.-N. GUO AND CH.-P. CHEN, Some completely monotonic functions involving the gamma and polygamma functions, J. Austral. Math. Soc., 80, (1) (2006), 81-88. RGMIA Res. Rep. Coll. 7, (1) (2004), Art. 5, 31-36. Available online at URL: http://rgmia.vu .edu .au/v7n1.html.

[17] F. QI, J. SÁNDOR, S. S. DRAGOMIR AND A. SOFO, Notes on the Schur-convexity of the extended mean values, Taiwanese J. Math. 9, (3) (2005), 411-420. RGMIA Res. Rep. Coll. 5, (1) (2002), Art. 3, 19-27. Available online at URL: http://rgmia.vu.edu.au/v5n1.html.

[18] F. QI, S.-L. XU, The function $\left(b^{x}-a^{x}\right) / x$ : Inequalities and properties, Proc. Amer. Math. Soc. 126, (11) (1998), 3355-3359.

[19] F. QI, SH.-Q. ZHANG, Note on monotonicity of generalized weighted mean values, R. Soc. Lond. Proc. Ser. A Math. Phys. Eng. Sci. 455, (1989) (1999), 3259-3260.

[20] ZH.-X. WANG, D.-R. Guo, Special Functions, Translated from the Chinese by D.-R. Guo and X.-J. Xia, World Scientific Publishing, Singapore, 1989.

[21] Zh.-X. Wang, D.-R. Guo, Téshū Hánshú Gáilùn (A Panorama of Special Functions), The Series of Advanced Physics of Peking University, Peking University Press, Beijing, China, 2000. (Chinese)

[22] D. V. WIDDER, The Laplace Transform, Princeton University Press, Princeton, 1941. 